\title{
Gynecologic Pain in Adolescents
}

\section{Kate McCracken, MD}

\author{
Address \\ Pediatric and Adolescent Gynecology, Nationwide Children's Hospital, 700 \\ Children's Drive, Columbus, OH, 43205-2664, USA \\ Email: kate.mccracken@nationwidechildrens.org
}

Published online: 8 July 2016

(C) Springer International Publishing AG 2016

\section{This article is part of the Topical Collection on Pediatric Gynecology}

Keywords Gynecologic pain - Adolescents - Dysmenorrhea - Endometriosis - Mullerian anomalies · Adnexal masses · Musculoskeletal pain

\section{Opinion statement}

Gynecologic pain affects a significant number of adolescents. While the differential diagnosis for such pain is often extensive, the treatment goals are the same-to relieve pain and minimize the effect it has on an adolescent's daily functioning, and to maximize their future reproductive potential. For management of acute gynecologic pain-such as in cases of adnexal torsion or obstructive anomalies-surgical intervention is often warranted. Pain related to more chronic processes-such as endometriosis-is treated both medically and surgically. Hormonal therapy (either combined estrogen/progestin or progestin-only) is the mainstay of medical therapy. Newer methods, such as the levonorgestrel intrauterine devices (LNG-IUD), show promise as a management option for chronic endometriosis-related pain. The LNG-IUD also provides highly effective long-acting reversible contraception, and is a first-line recommended contraceptive method for adolescents. Other etiologies of gynecologic pain may be infectious and are therefore treated by antibiotics. Providers must perform a thorough history and physical exam - with attention to patient confidentiality. Establishing a good rapport with both patients and their caregivers is crucial. Regular follow-up to assess treatment efficacy is necessary-as certain conditions may require a stepwise progression of treatment modalities for resolution of symptoms. While it is often challenging caring for an adolescent with chronic gynecologic pain, it is also extremely important and rewarding. Optimizing the gynecologic health of adolescent females will allow them to function at their full potential and lead productive, enjoyable lives.

\section{Introduction}

Gynecologic pain in adolescents is common, and patients may present to a variety of care settings-primary care providers, gynecologists, urologists, gastroenterologists, emergency care providers, and pediatric surgeons. Gynecologic pain in adolescents may have multiple etiologies-these are listed in Table 1. The pain 
Table 1. Acute and chronic causes of gynecologic pain in adolescents (adapted from Laufer M. gynecologic pain: dysmenorrhea, acute and chronic pelvic pain, endometriosis, and premenstrual syndrome. In: Emans SJ, Laufer Mr. Emans, Laufer, Goldstein's pediatric and adolescent gynecology. $6^{\text {th }}$ ed. Philadelphia, PA: Lippincott Williams and Wilkins; 2012:239-271.) [4]

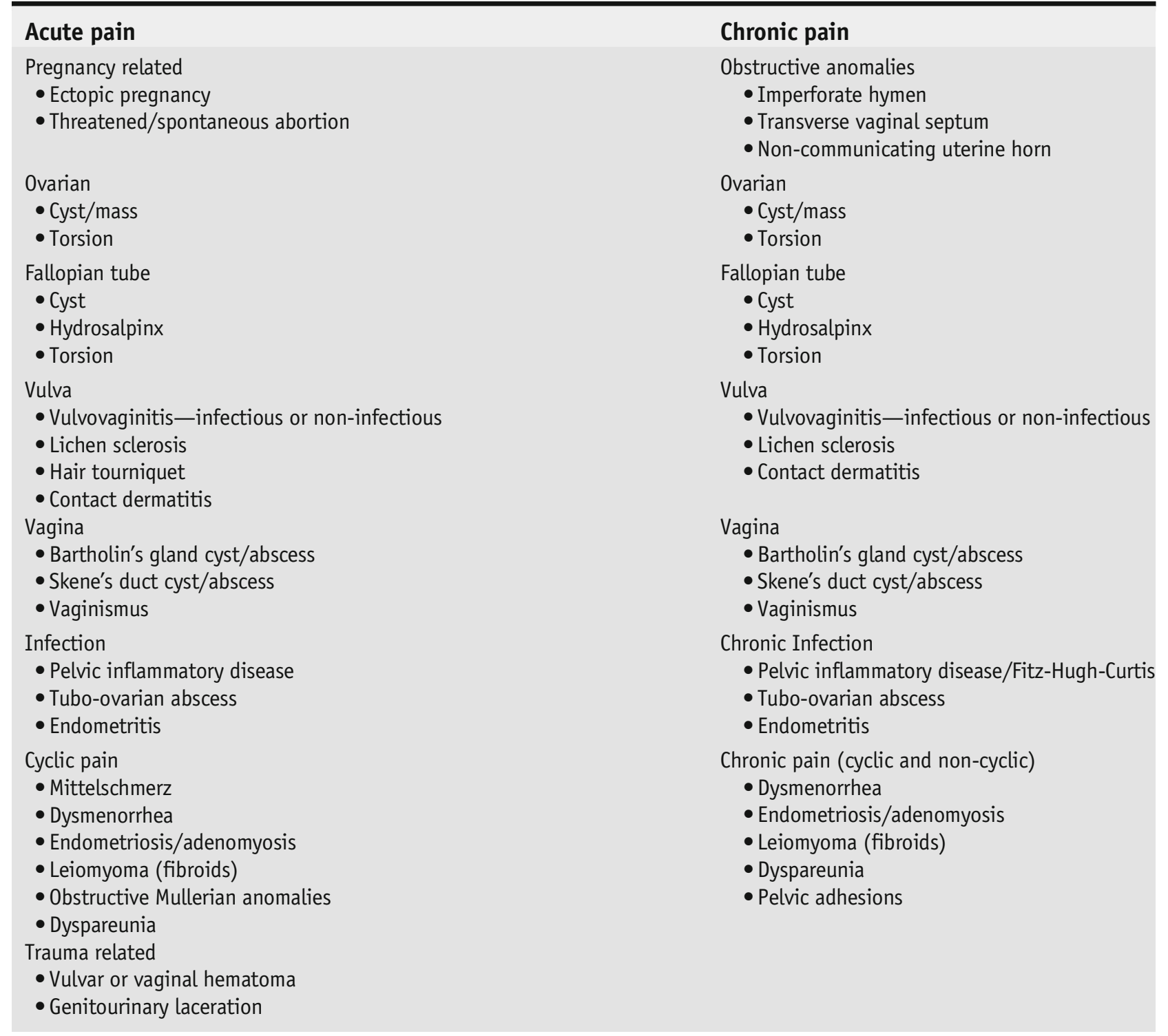

may be primary (not attributed to pathology) or secondary (attributed to a pathologic condition); it may be acute or chronic in nature, and cyclic or continuous. Practitioners must also consider and rule out other nongynecologic etiologies of pain-such as gastrointestinal, genitourinary, musculoskeletal, neurologic, or psychologic conditions. While the differential diagnosis is extensive, a thorough and multisystem evaluation of the pain will lead the provider to an accurate diagnosis, and help dictate the appropriate treatment.

Treatment of gynecologic pain is varied and may require a stepwise progression of treatment and/or 
multiple modalities. Treatment is dictated by the diagnosis. Currently, treatment may include medication, surgical interventions, physical therapy, trigger point injections, behavioral and psychological therapy, and complementary/alternative medicine. There may also be scenarios in which observation and expectant management are the treatment of choice. Timely and accurate management of gynecologic pain is crucial to avoid significant negative impact on quality of life and reproductive health of the adolescent [1].

When initially evaluating an adolescent with pain, establishing a rapport is paramount. Patient-parent dynamics, parental involvement, and maintaining confidentiality are issues that must be navigated by the provider. Obtaining a thorough history is the initial step in evaluating pain-specifically, providers should elicit information about the onset, duration, timing, character, quality, location, and radiation of the pain. Any aggravating, relieving, and associated symptoms should be documented. It is crucial to obtain a gynecologic history, and when applicable, a confidential sexual and psychosocial history (including substance use/abuse and sexual or physical abuse). Asking what the patient attributes her pain to offers insight into the patient's developmental stage, coping mechanisms, and perhaps even confidential history components. How an adolescent responds to and copes with pain may be complicated by psychosocial and developmental changes that accompany adolescence [2]. Often, the patient may be hesitant to discuss their pain, as they may view discussing gynecologic, urologic, or gastroenterological concerns as embarrassing. Providers should also assess the impact of pain on the patient's activities of daily life-school attendance and performance, extracurricular activity involvement, sleep, $\mathrm{mood} /$ behavior, and family life. It is important to explore and document the timeline and response to any prior therapies. Often, the patient has had multiple visits for complaints of pain and may have been previously treated in some manner. A past medical and surgical history, in addition to any relevant family history, should be documented. Lastly, asking the adolescent and her caregiver to indicate their goals of therapy will help ensure that the provider and patient have common goals.

The physical exam will be tailored by the differential diagnosis created after obtaining a history. It should also take into account the patient's age, maturity level, and sexual activity status. Reassuring the patient that she has the right to stop the physical exam at any point provides the adolescent a sense of control and helps solidify the rapport established at the beginning of the encounter.

Key elements of the physical exam include the following: review of vital signs, height/weight (and if possible growth charts), Tanner staging of breasts and pubic hair, an abdominal exam (note tenderness, masses, scars, hernias), a musculoskeletal exam (noting trigger points, posture, scoliosis, leg length discrepancies), an external genital exam (noting vulvar lesions, congenital anomalies, hymenal anatomy, discharge, trauma, tenderness of the vulva/perineum/introitus), and if applicable, an internal genital exam. A speculum exam is useful to evaluate vaginal patency, the vaginal mucosa and cervix, and obtain cultures if there is concern for an infectious process. A bimanual exam is a means to evaluate uterine and adnexal size, shape, presence of masses, and tenderness. If a speculum and/or bimanual exam are not feasible, a lubricated cotton swab may be used to assess vaginal patency, and a rectoabdominal exam may be used to assess the rectovaginal septum and posterior cul-de-sac. Often, a rectoabdominal exam is better tolerated than a bimanual exam in the young and non-sexually active adolescent.

Further diagnostic testing may include the following: pelvic ultrasound, pelvic magnetic resonance imaging (MRI), vaginal cultures, labs, and menstrual calendars. A pregnancy test must be performed in sexually active adolescents. Pelvic ultrasound is helpful if the provider is unable to perform a pelvic exam or if they suspect a mass or congenital anomaly. Pelvic MRI is a particularly useful tool in evaluating congenital anomalies of the reproductive tract. Diagnostic laparoscopy is commonly used to confirm a diagnosis and provide treatment of certain conditions.

\section{Treatment}

As previously mentioned, the differential diagnosis of gynecologic pain in the adolescent can be extensive-as can the corresponding treatment options. The course of treatment is dictated by the suspected diagnosis, and in some cases, providers may move through several modalities before the desires treatment 


\section{Pharmacologic treatment}

effect is obtained. We will begin our discussion of treatment options with pharmacologic therapies.

\section{Non-steroidal anti-inflammatory medications}

Initial therapy for dysmenorrhea-severe cramping lower abdominal pain that occurs during and/or prior to menses-is typically non-steroidal anti-inflammatory drug (NSAID). NSAIDs inhibit prostaglandin synthesis. They have both analgesic and anti-inflammatory properties. A meta-analysis of 63 randomized controlled trials found that NSAIDs were significantly more effective for pain relief than placebo [3]. Typically, patients are instructed to take NSAIDs on an as-needed basis when they experience dysmenorrhea. However, in some patients, it may be beneficial to schedule dosing-starting at the onset of menses and continuing as long as they experience cramping. Commonly used NSAIDs include ibuprofen, naproxen, and mefenamic acid. The response to NSAIDs is variable, and lack of response to one NSAID should prompt the provider to prescribe an alternate NSAID [4]. Patients should be counseled that side effects may include gastrointestinal irritation, heartburn, nausea, vomiting, headache, and allergic reactions. Adolescents with known/suspected peptic ulcer disease, GI bleeding, clotting disorders, renal disease, or known allergies to this class of medication should avoid NSAID use.

\section{Combined hormonal contraceptives}

Combined hormonal contraceptives (CHCs) are a mainstay of treatment in cases of primary dysmenorrhea and secondary dysmenorrhea-especially in the case of endometriosis. They are also used for ovulation suppression to reduce the frequency of physiologic ovarian cysts.

CHCs inhibit ovulation by suppression gonadotropin secretion. Specifically, the progestin component in the pill suppresses the luteinizing hormone (LH) surge that is necessary for ovulation, while the estrogen component suppresses follicle-stimulating hormone (FSH) and therefore, suppresses follicular development. Estrogen also potentiates the action of progestin and stabilizes the endometrium [5]. The proposed mechanism of action for treatment of endometriosis with a CHC is decidualization followed by atrophy of the endometrial tissue [6].

Most adolescents are overall healthy and will not have contraindications to the use of CHC. However, it is imperative to review the patient's past medical history and family history for any possible contraindications to an estrogencontaining pill prior to initiation. The Centers for Disease Control (CDC) has compiled a useful guide outlining the medical eligibility criteria for contraceptive use [7].

Combined hormonal therapy is the first-line treatment of endometriosis. CHCs may be administered in a cyclic or continuous fashion. Typically, patients with endometriosis have greater reduction in their symptoms with a continuous CHC. A progestin-dominant monophasic pill is preferred [4].

It is important to note that postoperative management of endometriosis in an adolescent is crucial for pain control, ensuring participation in school and activities, and preservation of fertility. The importance of treating endometriosis in adolescents cannot be overstated-as studies have shown that early diagnosis 
and treatment (both medically and surgically) decrease disease progression, and without treatment, endometriosis will progress $[8,9]$.

As mentioned above, CHCs are also frequently used for patients with a history of benign functional cysts. While typical low-dose ( $\leq 35 \mu \mathrm{g}$ ethinyl estradiol) may not be as protective against benign ovarian cysts compared to the original 50- $\mu \mathrm{g}$ EE pills-studies do show decreased formation of functional cysts $>3 \mathrm{~cm}$ with monophasic pills [10]. It is important to note that CHCs do not help resolve an already formed ovarian cyst.

Progestins may also be used for treatment of dysmenorrhea and endometriosisrelated pain. Commonly used progestins include norethindrone acetate (5$15 \mathrm{mg}$ orally per day), depo-medroxyprogesterone acetate (150 mg intramuscularly every 12 weeks), and the levonorgestrel intrauterine device (delivers $20 \mu \mathrm{g}$ /day over 5 years).

Given the potential side effects of progestin therapy-irregular menstrual bleeding, weight gain, acne, bloating, and emotional ability_-progestin therapy is often used in patients who have contraindications to combined hormonal contraception. Patients using depo-medroxyprogesterone acetate (DMPA) should be counseled that the U.S. Food and Drug Administration has issued a "black box warning" - that DMPA should be used as a long-term birth-control method (e.g., longer than 2 years) only if other birth control methods are inadequate" [5]. It is important to note that the loss in bone mineral density is most rapid within the first 2 years of use, then slows, and bone density is restored within $<3$ years after the discontinuation of DMPA. Furthermore, there is no need to obtain bone mineral density testing in adolescents using DMPA. Providers should weigh the risks and benefits of DMPA use and counsel patients electing to use DMPA on the need for adequate calcium and vitamin D intake.

Recently, there has been increased research on the utility of the levonorgestrel intrauterine device (LNG-IUD) for the management of endometriosisrelated pain $[11,12]$. The LNG-IUD has the advantages of avoiding compliance with a daily medication and offering highly efficacious long-acting reversible contraception. Studies have randomized women with known endometriosis to LNG-IUD or gonadotropin-releasing hormone agonists, and both treatments were found to be effective for pain control [13, 14], while the LNG-IUD had higher continuation rates and lower pain scores at 3 years [15]. Future research evaluating the efficacy of LNG-IUD for adolescents with gynecologic pain is needed.

Gonadotropin-releasing hormone agonists

Second-line therapy for endometriosis consists of gonadotropin-releasing hormone agonists (GnRHa) with add-back therapy (typically an oral progestin). GnRHa bind to pituitary receptors which results in the down-regulation of the pituitary-ovarian axis and a reversible hypoestrogenic state-which in turn results in amenorrhea and endometrial atrophy. These medications have been well studied, and their use in adolescents is supported [4].

GnRHa therapy is available in injectable forms, nasal spray, and implants. Commonly used doses include depot leuprolide $11.25 \mathrm{mg}$ administered every 3 months or depot leuprolide $3.75 \mathrm{mg}$ every 4 weeks. 
Because a stimulatory phase occurs prior to the down-regulation of the pituitary-ovarian axis, patients should be counseled to expect a "flare" in symptoms accompanied by a withdrawal bleed approximately 21-28 days after administration of the initial GnRHa dose.

Commonly reported side effects are related to the hypoestrogenic state-hot flushes, vaginal dryness, decreased libido, mood swings, and bone mineral depletion. Given the concern about decrease in bone density, the FDA has limited the use of GnRHa to courses not lasting longer than 6 months [4]. The use of GnRHa in adolescents is of concern, given that adolescence is a period of normal bone acquisition. For that reason, GnRHa therapy is not recommended for patients younger than 16 years $[16,17]$.

The goal of add-back therapy is to reduce the negative effects of GnRHa therapy (i.e., bone density loss and hot flushes) without stimulating endometriosis growth and related pain. The utility of add-back therapy lies within the principle of an "estrogen threshold hypothesis"- that the amount of estrogen necessary to prevent bone loss, hot flushes, and other hypoestrogenic symptoms is less that the amount of estrogen that would stimulate endometriosis [18]. Add-back therapy typically consists of norethindrone acetate $5 \mathrm{mg}$ daily or conjugated estrogens (0.625 mg) taken with medroxyprogesterone acetate $10 \mathrm{mg}$ or norethindrone acetate $5 \mathrm{mg}$ daily. Optimization of calcium and vitamin D is also important.

\section{Antibiotic therapy}

In cases of pelvic inflammatory disease (PID) and tubo-ovarian abscesses, antibiotic therapy is the mainstay of treatment. PID is a common etiology of infectious morbidity in adolescent females. PID is an acute inflammatory infection of the upper genital tract in women-which may involve the uterus, fallopian tubes, ovaries, and other pelvic structures. If untreated, it may result in significant sequelae - chronic pelvic pain, pelvic adhesive disease, increased risk of ectopic pregnancy, and increased risk of infertility. Endometritis, salpingitis, tubo-ovarian abscesses, and pelvic peritonitis may occur in the setting of PID.

PID antibiotic therapy includes broad-spectrum coverage, while Neisseria gonorrhoeae and Chlamydia trachomatis are commonly implicated in PID cases; multiple microorganisms that comprise vaginal flora have been associated with PID [19]. Outpatient PID therapy is typically appropriate-unless the patient meets any of the following criteria (Table 2) - in which case, inpatient management is suggested.

Of note, there is no evidence that adolescents benefit from hospitalization for PID treatment-the decision to hospitalize or not should be based upon the clinical picture, not the patient's age.

Table 2. Indications for inpatient PID treatment (modified from Workowski KA, Bolan GA [44])

Surgical emergency cannot be excluded (i.e., appendicitis)

Pregnant patient

Failed outpatient oral antibiotic therapy

Patient unable to comply or tolerate outpatient oral antibiotic therapy

Severe illness-nausea/vomiting, high fever

Presence of a tubo-ovarian abscess 


\section{Surgery}

It is important to counsel adolescents to notify their partners if possible, to encourage partner testing/treatment, and to abstain from sexual intercourse until therapy is completed, their symptoms have resolved, and their partners have been treated. Patients should be offered testing for other sexually transmitted infections-in particular HIV. Patients should have close follow-up (ideally within 3 days of initiating oral outpatient therapy). Adolescents may be at increased risk for poor adherence to the CDC recommendations [20]. Assessment of patient safety, any forced intercourse, access to condoms, and access to contraceptive methods should occur in a confidential manner.

Recommended treatment regimens for outpatient and inpatient therapy are listed below in Table 3 .

\section{Laparoscopy}

Laparoscopy is useful in both establishing the diagnosis and providing a means of treatment for gynecologic pain. In addition, laparoscopy is associated with decreased morbidity and shorter length of hospital stays compared to laparotomy [21, 22].

The management of adnexal masses-both benign and malignant-is beyond the scope of this paper. Adnexal masses may be an etiology for both acute and chronic gynecologic pain; thoughtful preoperative assessment and risk stratification is necessary to determine the safest and most effective surgical treatment course. Given the low risk of malignancy in the pediatric and adolescent population, in the majority of cases, laparoscopy is a wise choice, and the gold standard for the management of adnexal masses. In many patients with adnexal cysts, a laparoscopic procedure is not emergent-however, in cases of a ruptured hemorrhagic ovarian cyst if the patient is hemodynamically unstable, emergent surgical intervention should be undertaken. Hemoperitoneum is not a contraindication to laparoscopy [4].

Other indications for laparoscopy include the following: progressive dysmenorrhea, dysmenorrhea unresponsive to medical therapy, suspected endometriosis, or suspected chronic PID [23].

If an adolescent has not responded to treatment with NSAIDs and estrogen/ progestin or progestin-only therapy after 3-6 cycles of pills, and pain is adversely affecting her quality of life, then further assessment is needed [24]. Laparoscopy is the cornerstone of diagnosing endometriosis. Any suspicious lesions should be biopsied. It is important that a surgeon well versed in recognizing and treating adolescent endometriosis performs the laparoscopy. Endometriosis lesions in adolescents may appear differently than in adults. Specifically, adolescents are more likely to have red lesions, clear vesicles, white implants, small hemorrhagic peritoneal spots, or peritoneal defects/windows, rather than "classic" brown, black, or "powder burn" lesions that are seen in adults [4]. The "non-classic" lesions may be best visualized by placing the laparoscope within millimeters of the peritoneum or filling the pelvis with saline and placing the laparoscope "under water." While the optimal surgical technique for treating endometriosis has not yet been established, the data does indicate the women with symptoms of pelvic pain who have visible endometriosis at the time of laparoscopy should be treated. Furthermore, women with endometriomas should have complete excision of the endometrioma cyst wall, 


\section{Table 3. CDC recommendations for PID treatment [44]}

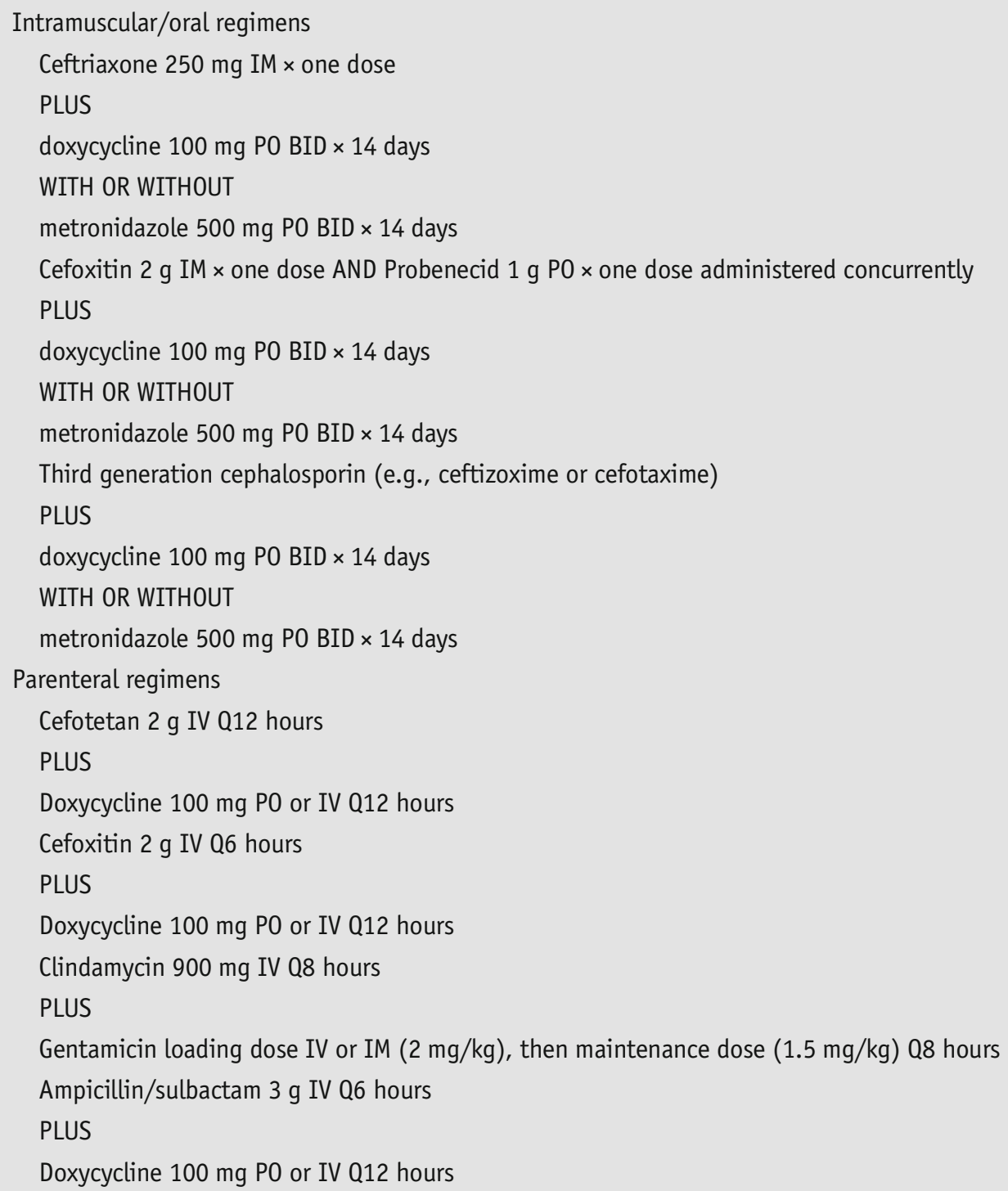

as there is a lower risk of recurrence with cyst wall excision compared to fenestration and ablation of the cyst wall [6]. All adolescents with endometriosis should be treated medically after surgical intervention. This will reduce the progression of symptoms and avoid recurrence.

In cases of suspected adnexal torsion, urgent surgical intervention is the mainstay of therapy. With the rotation of the ovary or fallopian tube on its pedicle, blood supply is compromised; this leads to venous congestion, impaired arterial supply, ischemia, pain, and if untreated, necrosis and loss of the adnexa [25]. Patients typically present with acute onset of pain with associated nausea and vomiting. Adnexal masses-such as ovarian or paratubal cyst-may predispose the patient to adnexal torsion. Normal ovaries may also torse. Standard 
laparoscopic surgical technique is used in cases of suspected adnexal torsion. Recent data has indicated that an emphasis on detorsion of the adnexa and ovarian preservation, rather than removal of adnexal structures, is the standard of care [4]. Fertility-sparing procedures are of the utmost importance in adolescents. In some cases, it is useful to make an incision in the ovarian cortex to essentially bivalve the ovary and decrease the intraovarian pressure [26].

Laparoscopy is also useful in the removal of non-communicating uterine horns that contain functional endometrium. The shorter hospital stay, reduced postoperative pain, and improved cosmesis make laparoscopy an attractive option. In patients with mullerian anomalies-and uterine remnants-laparoscopy is a safe and effective means for removal if careful surgical consideration is given to the patient's unique anatomy (i.e., associated urinary tract anomalies, possible endometriosis, and adhesive disease) [27].

In cases of chronic pelvic inflammatory disease or non-resolving tubo-ovarian abscesses, surgical intervention may be undertaken. Laparoscopy may be useful in patients who have repeatedly been diagnosed with PID, but who have not seemed to respond to standard treatment. In such cases, evaluation for other etiologies of pain, such as endometriosis, may be found during laparoscopic evaluation. Laparoscopy may also be used for management of PID sequelae such as pelvic adhesions. Pelvic adhesions may result from etiologies other than PID; they may be present in cases of endometriosis, secondary to prior surgical procedures or from ruptured appendicitis. Adhesions do not always produce pain. The data is mixed on whether lysis of adhesions provides therapeutic benefit $[2,28]$. In some cases of non-resolving tubo-ovarian abscesses, interventional radiology placement of a pelvic drain may be useful.

\section{Surgical procedures for obstructive anomalies}

Patients with an obstructive anomaly, such as an imperforate hymen, transverse vaginal septum, obstructed hemivagina, distal vaginal atresia, vaginal agenesis, or non-communicating functional uterine horn, may present with either acute or chronic pain related to obstructed menstrual outflow and development of hematometra or hematocolpos. In cases of an imperforate hymen or transverse vaginal septum, patients may also present with acute urinary retention. Primary amenorrhea with abdominal or pelvic pain should raise concern for an obstructive anomaly. Additionally, in patients with menstrual flow, but progressive or non-responsive dysmenorrhea, a partial obstruction must be considered. The diagnosis is often delayed in these cases [29]. Accurate diagnosis of an obstructive anomaly is essential-as there is a difference in surgical management techniques for imperforate hymen versus transverse vaginal septum versus distal vaginal atresia. MRI is useful in delineating the anatomy pre-operatively.

An imperforate hymen with symptomatic hematocolpos is usually managed emergently. While under anesthesia, excision of excess hymenal tissue is performed, the hematocolpos is drained, and the patient experiences immediate pain relief. Once excised, hymenal tissue does not regenerate; thus, there is no risk of stenosis.

In cases of transverse vaginal septa, the first consideration is the level of the septum within the vagina (low, middle, high). Second, the thickness of the septum must be established. MRI is a useful tool for both pieces of information. If the septum is thin, it may be resected, and the vaginal mucosa is then reapproximated primarily. However, in cases of thick septa, the reapproximation of vaginal mucosa 
is more challenging. If the upper vagina is well distended, it may be possible to mobilize normal upper vaginal mucosa so that it can be brought together with the distal vaginal mucosa without the anastomosis being under undue tension. However, if this is not possible, other techniques may be utilized, such as using the vaginal septum to make flaps for a bridge of vaginal tissue between the upper and lower vagina $[29,30]$. Vaginal stenosis is common after resection of transverse vaginal septa-thus, vigilant surveillance for this complication and liberal use of vaginal dilators is important.

A challenging case may be that of the obstructed hemivagina. In this case, the patient typically has a didelphic uterus with duplicated cervices and septal tissue obstructing menstrual flow from one cervix. This results in hematometrocolpos and cervical distension on the obstructed side. Complete resection of the septal tissue without damage to the distended cervix is crucial. Once the septum is excised, the vaginal mucosa is reapproximated in an interrupted fashion. There are some cases in which an obstructed hemivagina is present and there is a microperforation in the tissue-this is challenging because the septum may not be distended and the boundaries of the septum are difficult to discern. Stay sutures or a catheter threaded into the microperforation and pulled downward is helpful to provide traction so that the septum can be resected without damage to the cervix above [29, 30].

Distal vaginal atresia refers to cases in which an upper vagina is present, but the distal vagina has not developed and instead is replaced by fibrous tissue. It is important to distinguish this case from that of a thick low transverse vaginal septum. With distal vaginal atresia, a vaginal pull-through is performed. In this procedure, dissection of the vesicovaginal and rectovaginal spaces is performed to locate the upper vagina; then, the normal vaginal mucosa is mobilized to the perineum to join the upper vagina to the lower vaginal dimple [29, 31, 32].

Treatment of obstructive anomalies is important as there are higher rates of endometriosis, hematosalpinx, pelvic inflammatory disease, and adhesive disease related to menstrual backflow in these adolescents [29].

\section{Incision, drainage, and word catheter use}

Vulvar abscesses do occur in adolescent patients. Conservative therapy with antibiotics may be considered; however, in many cases, a surgical incision and drainage of the abscess is necessary. Standard technique is used in which the fluctuant area of the abscess is identified and a scalpel is used to make in incision into the abscess cavity, followed by irrigation of the abscess and mechanical destruction of any loculations. A culture of the purulent abscess contents should be obtained. Packing of the abscess and the use of a drain depend on the size of the abscess, location, and hemostasis. In adolescents, this procedure is usually performed under anesthesia, but in some situations, local anesthesia may be utilized. In cases of a Bartholin's gland cyst and/or abscess, a Word catheter is left in place for a period of time to promote epithelialization.

\section{Diet, lifestyle, and exercise}

Patients with dysmenorrhea should be encouraged to exercise regularly (goal $30 \mathrm{~min}$ of cardiovascular activity per day), make healthy food choices, and follow stress-reduction strategies. Adolescence is a stressful time, and poor sleep hygiene, unbalanced diets, and lack of regular exercise may exacerbate primary 


\section{Physical therapy}

or secondary dysmenorrhea. Providers should assess these lifestyle components and assist in making recommendations for behavioral changes as necessary.

Several complementary and alternative medicine therapies have been proposed as helpful for dysmenorrhea symptoms. However, randomized controlled trials and studies with adolescent cohorts are limited. Dong Quai, evening primrose oil, and chaste tree berry are commonly marketed for relief of menstrual discomfort. Data is mixed on their efficacy [33]. Some studies show that following a low-fat diet and consuming a fish oil supplement may relieve dysmenorrhea symptoms [33].

Acupuncture has been well studied and appears to reduce pain in both primary dysmenorrhea and endometriosis patients [1, 4, 34-37]. Adolescents may benefit from acupuncture therapy from a trained, certified provider.

Lastly, yoga may be beneficial for dysmenorrhea symptoms. A randomized controlled trial of 92 adolescents found that practicing yoga reduced both the severity and duration of primary dysmenorrhea compared to controls [38].

\section{Other treatments}

In cases of musculoskeletal related pain, physical therapy may be beneficial. Musculoskeletal dysfunction may occur as a response to a primary gynecologic problem, or it may be the primary etiology of a patient's pain [2]. A discussion on musculoskeletal pain is beyond the scope of this article. However, it is important to note that musculoskeletal pain must be evaluated and treated. Physical therapy-with an emphasis on pelvic floor physical therapy-is an effective treatment for musculoskeletal pain. Physical therapy is also useful in the treatment of adolescent vulvodynia, vaginismus, and endometriois $[1,4$, $37,39]$. The use of biofeedback and self-vaginal dilators may be combined with pelvic floor physical therapy. While this may be challenging in adolescents, the treatment is effective. In a retrospective review of adolescents with musculoskeletal pain who presented to a pediatric and adolescent gynecology practice, physical therapy resulted in resolution of symptoms in $95 \%$ of patients [40].

Topical heat

Heat is a commonly used therapeutic measure. This holds true for adolescents with dysmenorrhea [41]. Low-dose heat application has been shown to reduce dysmenorrhea $[42,43]$. Heat vasodilates and thus, increases smooth muscle perfusion and relaxation. This leads to a decreased perception of pain. Heat is a non-invasive, cost-effective, and low-risk adjunct to other therapeutic measures.

\section{Conclusion}

Gynecologic pain in adolescents is a common problem. Obtaining a complete history and physical allows the provider to develop a differential diagnosis. The suspected diagnosis will guide therapy. Patients with chronic pain may require regular visits. It is important to validate the patient's pain, offer reassurance when dangerous pathology has been ruled out, and set realistic treatment goals 
[37]. Encouraging the patient to attend school and participate in normal activities is crucial.

As with any treatment modality, future research should focus on welldesigned randomized controlled prospective trials to help establish the most efficacious, safe, minimally invasive, and cost-effective option. Treatment of gynecologic pain in adolescents ensures that our patients' reproductive health is maximized and that they can participate fully in life.

\section{Compliance with Ethical Standards}

\section{Conflict of Interest}

The author declares that she has no conflict of interest.

\section{Human and Animal Rights and Informed Consent}

This article does not contain any studies with human or animal subjects performed by any of the authors.

\section{References and Recommended Reading}

1. Trotman GE, Gomez-Lobo V. Pelvic pain in the adolescent. Contemporary OB/GYN. 2013;58(1):50-5.

2. Song AH, Advincula AP. Adolescent chronic pelvic pain. J Pediatr Adolesc Gynecol. 2005;18(6):371-7.

3. Marjoribanks J, Proctor MS, Farquhar C. Nonsteroidal anti-inflammatory drugs for primary dysmenorrhea. Cochrane Database Syst Rev. 2003;4:CD001751.

4. Laufer M. Gynecologic pain: dysmenorrhea, acute and chronic pelvic pain, endometriosis, and premenstrual syndrome. In: Emans SJ, Laufer M, editors. Emans, Laufer, Goldstein's pediatric and adolescent gynecology. 6th ed. Philadelphia, PA: Lippincott Williams and Wilkins; 2012. p. 239-71.

5. Dayananda I, Emans SJ, Goldberg A. Contraception. In: Emans SJ, Laufer M, editors. Emans, Laufer, Goldstein's pediatric and adolescent gynecology. 6th ed. Philadelphia, PA: Lippincott Williams and Wilkins; 2012. p. 447-73.

6. Pfeifer S, Reindollar R, Goldberg J, et al. Treatment of pelvic pain associated with endometriosis: a committee opinion. Fertil Steril. 2014;101(4):927-35.

7. Peterson HB, Curtis KM, Jamieson D, et al. U.S. medical eligibility criteria for contraceptive use, 2010. MMWR Recomm Rep. 2010;18(RR-4):1-86.

8. Doyle JO, Missmer SA, Laufer MR. The effect of combined surgical-medical intervention on the progression of endometriosis in an adolescent and young adult population. J Pediatr Adolesc Gynecol. 2009;22:25763.

9. Unger CA, Laufer MR. Progression of endometriosis in non-medically managed adolescents: a case series. J Pediatr Adolesc Gynecol. 2011;24:321-23.
10. Christensen JT, Boldsen JL, Westergaard JG. Functional ovarian cysts in premenopausal and gynecologically healthy women. Contraception. 2002;66(3):153-7.

11. Vercellini P, Frontino G, De Giorgi O, et al. Comparison of a levonorgestrel-releasing intrauterine device versus expectant management after conservative surgery for symptomatic endometriosis: a pilot study. Fertil Steril. 2003;80:305-9.

12. Abou-Setta AM, AlInany HG, Farquhar C. Levonorgestrel-releasing intrauterine device (LNGIUD) for symptomatic endometriosis following surgery. Cochrane Database of Systematic Reviews 2006, Issue 4. Art.No.: CD005072. DOI: 10.1002/14651858. CD005072.pub2.

13. Petta CA, Ferriani FA, Abrao MS, et al. Randomized clinical trial of a levonorgestrel-releasing intrauterine system and a depot GnRH analogue for the treatment of chronic pelvic pain in women with endometriosis. Hum Reprod. 2005;20(7):1993-8.

14. BayogluTekin Y, Dilbaz B, Altinbas SK, et al. Postoperative medial treatment of chronic pelvic pain related to severe endometriosis: levonorgestrel-releasing intrauterine system versus gonadotropin-releasing hormone analogue. Fertil Steril. 2011;95:492-6.

15. Petta CA, Ferriani RA, Abrao MS, et al. A 3-year followup of women with endometriosis and pelvic pain users of the levonorgestrel-releasing intrauterine system. Eur J Obstet Gynecol Reprod Biol. 2009;143(2):128-9.

16. Laufer MR, Sanfilippo J, Rose G. Adolescent endometriosis: diagnosis and treatment approaches. J Pediatr Adolesc Gynecol. 2003;16:S3-S11. 
17. Divasta AD, Laufer MR, Cm G. Bone density in adolescents treated with a GnRH agonist and add-back therapy for endometriosis. J Pediatr Adolesc Gynecol. 2007;20:293-7.

18. Barbieri RL. Endometriosis and the estrogen threshold theory: relation to surgical and medical treatment. J Reprod Med. 1998;43:297-2.

19. Ness RB, Kip KE, Hillier SL, et al. A cluster analysis of bacterial vaginosis-associated microflora and pelvic inflammatory disease. Am J Epidemiol. 2005;162:585-90.

20. Trent M. Status of adolescent pelvic inflammatory disease management in the United States. Curr Opin Obstet Gynecol. 2013;25(5):350-6.

21. Yeun PM, Yu KM, Yip SM, et al. A randomized prospective study of laparoscopy and laparotomy in the management of benign ovarian masses. Am J Obstet Gynecol. 1997;177(1):109-14.

22. Canis M, Rabischong B, Houlle C, et al. Laparoscopic management of adnexal masses: a gold standard? Curr Opin Obstet Gynecol. 2002;14(4):423-8.

23. Hewitt GD, Brown RT. Acute and chronic pelvic pain in female adolescents. Med Clin North Am. 2000;84(4):1009-25.

24. Laufer MR. Helping "adult gynecologists" diagnose and treat adolescent endometriosis: reflections on my 20 years of personal experience. J Pediatric Adolesc Gynecol. 2011;24:S13-7.

25. Quint EH, Johnson MA, Breech LL. Acute pelvic pain in adolescents. J Pediatr Adolesc Gynecol. 2003;16:253-5.

26. Styer AK, Laufer MR. Ovarian bivalving after detorsion. Fertil Steril. 2002;77(5):1053-5.

27. Will MA, Marsh CA, Smorgick N, et al. Surgical pearls: laparoscopic removal of uterine remnants in patients with Mayer-Rokitansky-Kuster-Hauser syndrome. J Pediatr Adolesc Gynecol. 2013;26:224-7.

28. Swank DJ, Swank-Bordewijk SC, Hop WC, et al. Laparoscopic adhesiolysis in patients with chronic abdominal pain: a blinded randomised controlled multicentre trial. Lancet. 2003;361(9365):1247-51.

29. Dietrich JE, Millar DM, Quint EH. Obstructive reproductive tract anomalies. J Pediatr Adolesc Gynecol. 2014;27:396-402.

30. Miller RL, Breech LL. Surgical correction of vaginal anomalies. Clin Obstet Gynecol. 2008;51:223-36.
31. Nguyen L, Youssef S, Guttman FM, et al. Hydrometrocolpos in neonate due to distal vaginal atresia. J Pediatr Surg. 1984;19:510-4.

32. Jessel RH, Laufer MR. Management of lower vaginal atresia in patient with a unicornuate uterus. J Pediatr Adolesc Gynecol. 2013;26:e21.

33. Wornham WL. Complementary and alternative medicine for gynecology patients. In: Emans SJ, Laufer M, editors. Emans, Laufer, Goldstein's pediatric and adolescent gynecology. 6th ed. Philadelphia, PA: Lippincott Williams and Wilkins; 2012. p. 539-43.

34. Helms JM. Acupuncture for the management of primary dysmenorrhea. Obstet Gynecol. 1987;69(1):51-6.

35. Smith CA, Zhu X, He L, Song J. Acupuncture for primary dysmenorrhea. Cochrane Database Syst Rev. 2011;1:CD007854.

36. Wayne PM et al. Japanese-style acupuncture for endometriosis-related pelvic pain in adolescents and young women: results of a randomized shamcontrolled trial. J Pediatr Adolesc Gynecol. 2008;21(5):247-57.

37. Damle LF, Gomez-Lobo V. Pelvic pain in adolescents. J Pediatr Adolesc Gynecol. 2011;24(3):172-5.

38. Rakhshaee Z. Effects of three yoga poses (cobra, cat and fish poses) in women with primary dysmenorrhea: a randomized clinical trial. J Pediatr Adolesc Gynecol. 2011;24:192-6.

39. Clare CA, Yeh J. Vulvodynia in adolescence: childhood vulvar pain syndromes. J Pediatr Adolesc Gynecol. 2013;24(3):110-5.

40. Schroeder B, Sanfilippo JS, Hertweck SP. Musculoskeletal pelvic pain in a pediatric and adolescent gynecology practice. J Pediatr Adolesc Gynecol. 2000;13(2):90.

41. O'Connell K, Davis AR, Westhoff C. Self-treatment patterns among adolescent girls with dysmenorrhea. J Pediatr Adolesc Gynecol. 2006;19(4):285-9.

42. Akin $\mathrm{M}$ et al. Continuous, low-level, topical heat wrap therapy as compared to acetaminophen for primary dysmenorrhea. J Reprod Med. 2004;49(9):739-45.

43. Potur DC, Komurcu N. The effects of local low-dose heat application on dysmenorrhea. J Pediatr Adolesc Gynecol. 2014;27(4):216-21.

44. Workowski KA, Bolan GA. Sexually transmitted diseases treatment guidelines, 2015. MMWR Recomm Rep. 2015;64(RR-03):1-137. 